\title{
Unexpected Neurological Symptoms of Ruxolitinib: A Case Report
}

\author{
Francesca Furia $^{a}$, d, Maria P. Canevini ${ }^{a, b}$, Augusto B. Federici ${ }^{\text {b c }}$, Maria C. Carraro ${ }^{c}$
}

\begin{abstract}
Ruxolitinib is a highly potent $J A K 2$ inhibitor approved for the treatment of myelofibrosis (idiopathic or post-polycythemia vera or postessential thrombocythemia) and, more recently, for polycythemia vera with an inadequate response to or intolerant of hydroxyurea. The most common adverse events of ruxolitinib include immunosuppression with an increased risk of reactivation of silent infections and increased non-melanoma skin cancer. The known neurological side effects of ruxolitinib are dizziness and headache, but no neurological paroxysmal episodes have been recorded. This report deals with an 80-year-old outpatient woman with polycythemia vera turned into myelofibrosis who experienced neurological episodes of hypoesthesia and weakness of right arm and leg during ruxolitinib treatment.
\end{abstract}

Keywords: Ruxolitinib; Polycythemia vera; Myelofibrosis

\section{Introduction}

Polycythemia vera (PV) is the most common Philadelphia chromosome negative (Ph-) myeloproliferative neoplasm (MPN) and is characterized by an increased red cell mass and presence of the JAK2 (V617F) mutation in nearly all cases. Many patients come to medical attention based on routine blood work; however, symptoms including palpable splenomegaly, pruritus (classically aquagenic), erythromelalgia, and fatigue are common [1]. The greatest contributor to morbidity and mortality in patients with PV is thrombosis, which can be arterial or venous and may occur in atypical sites such as splanchnic bed [2]. There is also progression to myelofibrosis (post-PV MF), which occurs in $4.9-6 \%$ at 10 years [3].

Myelofibrosis (MF), which can present as a primary dis-

Manuscript submitted April 21, 2020, accepted August 20, 2020

Published online October 1, 2020

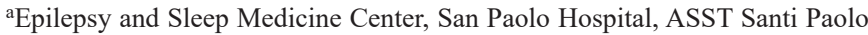
e Carlo, Milan, Italy

bUniversita degli Studi of Milan, Milan, Italy

${ }^{c}$ Hematology and Transfusion Medicine, Sacco Hospital of Milan, Milan, Italy ${ }^{\mathrm{d} C}$ Corresponding Author: Francesca Furia, Epilepsy and Sleep Medicine Center, San Paolo Hospital, ASST Santi Paolo e Carlo, Via Di Rudini 8, Milan 20142, Italy. Email: francesca.furia@gmail.com

doi: https://doi.org/10.14740/jh642 ease or can evolve from PV or essential thrombocythemia (ET), is characterized by progressive anemia, marrow fibrosis, and extramedullary hematopoiesis which becomes prominent especially in the spleen, with different degrees of splenomegaly. Constitutional symptoms (night sweats and weight loss), pruritus, fatigue, and sequelae of splenomegaly are common [4]. Apart from allogeneic stem-cell transplantation, which is the only curative treatment, only few therapies are available for the treatment of MF [5].

The main goal of therapy in PV is to prevent thrombotic events while avoiding iatrogenic harm and minimizing the risk of transformation to post-PV MF or acute myeloid leukemia (AML) [6].

Most patients receive low-dose aspirin and undergo phlebotomy [7], with goal of maintaining hematocrit values of less than $45 \%$. Aggressive treatment targeting a hematocrit of less than $45 \%$ lowers the risk of major thrombosis and death from cardiovascular causes [8].

Cytoreductive therapy is recommended in patients at high risk for thrombosis such as persistent or progressive hematologic abnormalities, splenomegaly, or symptoms and in patients who cannot undergo phlebotomy or who require frequent phlebotomies [6].

The most commonly used first-line cytoreductive agent is hydroxyurea. Some patients have an inadequate response to the drug or have unacceptable side effects at the doses required to consistently control hematocrit, platelet and white-cell count, splenomegaly and other symptoms. Many of these patients are classified as having resistance or intolerance to hydroxyurea according to European LeukemiaNet (ELN) criteria [9].

In patients who had an inadequate response or had side effects with hydroxyurea, ruxolitinib was superior to standard therapy in controlling the hematocrit, reducing the spleen volume, and improving symptoms associated with PV and MF [10].

Ruxolitinib, which is an orally bioavailable, potent and selective inhibitor of $J A K 2$, has been recently approved for the treatment of MF (idiopathic or post-PV or post-ET) and PV $[11,12]$.

The most common side effects of ruxolitinib include immunosuppression with an increased risk of reactivation of silent infections, especially herpes zoster (5.3 per 100 patient-years of exposure) and increased non-melanoma skin cancer (4.4 vs. 2.7 cases per 100 patient-years of exposure) [13]. There is also a concern about other infectious risks; however, this may be related to selection bias in the MF population rather than immunosuppressive properties of ruxolitinib itself $[14,15]$. 


\section{Case Report}

This report deals with an 80-year-old outpatient woman, who in 2012 was admitted for a hematological evaluation, at Sacco Hospital of Milan, for leukocytosis $\left(16.30 \times 10^{9} / \mathrm{L}\right)$ with neutrophilia $\left(12.37 \times 10^{9} / \mathrm{L}\right)$. Other laboratory data showed hemoglobin $15.6 \mathrm{~g} / \mathrm{dL}$, platelet count $458 \times 10^{9} / \mathrm{L}$ and lactate dehydrogenase (LDH) $228 \mathrm{U} / \mathrm{L}$. She referred aquagenic pruritus. The physical examination revealed spleen enlargement (the size of the spleen, as measured by ultrasonography, was $17 \mathrm{~cm}$ in length).

In her clinical history she was also known for thalassemia minor, gastric hemorrhage, hypothyroidism, hypertension and an episode of paroxysmal atrial fibrillation (PAF) in 2000. The PAF was treated with pharmacological cardioversion (propafenone), with return to sinus rhythm. It was evaluated from the cardiologist the possibility of an anti-coagulant treatment, but it was excluded because of the story of a gastric hemorrhage and an antiplatelet therapy has been started. The patient was also in therapy with levothyroxine, zofenopril, escitalopram, allopurinol, alendronic acid, calcium carbonate/ cholecalciferol and delorazepam.

She underwent genetic test with the result of mutation profile for $J A K 2(V 617 F)$ and a bone marrow biopsy that was diagnostic for PV.

The diagnosis of PV was made even if the criterium of hemoglobin was not satisfied according to the 2008 World Health Organization (WHO) diagnostic criteria [4] because of the presence of thalassemia minor.

The patient was treated with hydroxyurea $(2.5 \mathrm{~g} /$ week that was progressively increased to $5 \mathrm{~g} /$ week) with reduction of hemoglobin, but without any effect on splenomegaly; in fact an abdominal computed tomography (CT), performed in December 2017, revealed splenomegaly with longitudinal diameter of $21 \mathrm{~cm}$.

In October 2018, due to further spleen enlargement (23 $\mathrm{cm}$ of longitudinal axis), a bone marrow biopsy was performed again and an evolution in post-PV MF was confirmed.

According to the LeukemiaNet Consensus Group, the failure of hydroxyurea in reducing massive splenomegaly allows to define the patient resistant to this drug [9]; the resistance to hydroxyurea is one of the criteria to switch from this drug to ruxolitinib [10].

Consequently, hydroxyurea was stopped and ruxolitinib started $(10 \mathrm{mg} /$ die $)$. After 2 weeks the patient referred a complete resolution of pruritus, but it was observed an increase of hematocrit (49\%), so the dosage of ruxolitinib was augmented to $20 \mathrm{mg} /$ die. After 3 weeks of treatment, and with a hematocrit value of $40 \%$, the patient experienced episodes of hypoesthesia and weakness of the right arm (from the shoulder to the hand), which lasted from $10 \mathrm{~min}$ to $2 \mathrm{~h}$ and occurred several times a week, and in one occasion there was also hypoesthesia of the right leg. There was never loss of consciousness, neither hypertonia nor dystonia. Because of the described symptoms, the treatment with ruxolitinib was interrupted and hydroxyurea was restarted. When treatment with ruxolitinib was stopped, the patient did not experience any other neurological episodes.
A cerebral magnetic resonance imaging (MRI) hence ruled out an infection and revealed only millimetric focal areas characterized by fluid-attenuated inversion recovery (FLAIR) hyperintensity, without contrast enhancement, in correspondence of the periventricular white matter, suggestive for chronic cerebral vasculopathy. A Sovra-Aortic Trunks Echo-ColorDoppler did not reveal any significant stenosis and an echocardiography showed ejection fraction (FE) $55 \%$, mild atrial dilation and mild mitral and tricuspid valve insufficiency. The patient underwent the first neurological evaluation due to the suspicion of epileptic seizures versus transient ischemic events. At the neurological examination, the patient was oriented in the space-time parameters and asymptomatic for neurological symptoms. Cranic nerves were normal. There was no evidence of stenic, sensibility or cerebellar deficit. Standing and walking were normal.

Because there was no evidence of abnormalities at the neurological exams, in order to control the splenomegaly, the treatment with ruxolitinib was restarted at a lower dose ( $5 \mathrm{mg} /$ die $)$. But after 1 month, the neurological episodes reappeared, and the drug was definitively stopped with disappearance of neurological symptoms.

In October 2019, she had a neurological evaluation at the Epilepsy Center of San Paolo Hospital of Milan. The neurological examination was normal and an electroencephalography (EEG) performed in our center in January 2020 did not show any significant abnormalities.

\section{Discussion}

Until now, the only reported neurological common side effects of ruxolitinib are dizziness and headache [16].

In our patient it seems that ruxolitinib has been a dosageindependent trigger for neurological symptoms (the episodes started when she began the therapy and stopped when the treatment was interrupted), but the etiology of the episodes remains unclear. They could be interpreted as epileptic seizures, even if the clinical characteristics, especially the length, are not suggestive for seizures, or as transient cerebrovascular events, even if the frequency of the episodes is not typical for ischemic attacks.

The performed exams (brain MRI, Sovra-Aortic Trunks Echo-Color-Doppler and echocardiogram) did not reveal significant abnormalities and did not support the diagnosis of transitory ischemic attacks.

The clinical characteristics of the episodes are not clearly suggestive for epileptic seizures, especially considering the length (sometimes the symptoms lasted for $2 \mathrm{~h}$ ), but this diagnosis cannot be excluded because a video-EEG during the episodes was not performed. This exam could provide a correlation between the clinical symptoms and possible electrical cerebral modifications, supporting or not the diagnosis of epileptic seizures.

When the patient was evaluated at the Epilepsy Center, she was not taking ruxolitinib and she did not experience neurological episodes since more than 2 months. In order to rule out the diagnostic hypothesis of epileptic seizures, it would be 
necessary to perform a video-EEG during the symptoms. Considering that the episodes occurred only during the assumption of ruxolitinib, we should have restarted the drug in order to observe if the symptoms would reappear, allowing us to perform a video-EEG during them. We decided that it was unethical to restart ruxolitinib to see if effectively the drug was again associated with the neurological episodes and so performed a video-EEG.

Further investigations should be carried out in order to define the real effects of the drug on the Central Nervous System and it would be important that, if similar symptoms are observed in other patients, a video-EEG should be performed to define the nature of the episodes, helping in the management of the treatment of these patients.

\section{Acknowledgments}

None to declare.

\section{Financial Disclosure}

None to declare.

\section{Conflict of Interest}

None to declare.

\section{Informed Consent}

Not applicable.

\section{Author Contributions}

FF wrote the manuscript, reviewed the literature and took care of the patient. MPC and ABF supervised the manuscript. MCC gave suggestion to write manuscript and took care of the patient.

\section{Data Availability}

The authors declare that data supporting the findings of this study are available within the article.

\section{References}

1. Tefferi A. Polycythemia vera and essential thrombocythemia: 2012 update on diagnosis, risk stratification, and management. Am J Hematol. 2012;87(3):285-293.

2. Sekhar M, McVinnie K, Burroughs AK. Splanchnic vein thrombosis in myeloproliferative neoplasms. Br J Hae- matol. 2013;162(6):730-747.

3. Cerquozzi S, Tefferi A. Blast transformation and fibrotic progression in polycythemia vera and essential thrombocythemia: a literature review of incidence and risk factors. Blood Cancer J. 2015;5:e366.

4. Swerdlow SH, Campo E, Harris NL, Jaffe ES, Pileri SA, Stein $\mathrm{H}$, et al. WHO classification of tumours of haemopoietic and lymphoid tissues. Lyon, France IARC Press. 2008.

5. Tefferi A. Essential thrombocythemia, polycythemia vera, and myelofibrosis: current management and the prospect of targeted therapy. Am J Hematol. 2008;83(6):491-497.

6. Vannucchi AM, Kiladjian JJ, Griesshammer M, Masszi T, Durrant S, Passamonti F, Harrison CN, et al. Ruxolitinib versus standard therapy for the treatment of polycythemia vera. N Engl J Med. 2015;372(5):426-435.

7. Landolfi R, Marchioli R, Kutti J, Gisslinger H, Tognoni G, Patrono C, Barbui T, et al. Efficacy and safety of low-dose aspirin in polycythemia vera. N Engl J Med. 2004;350(2):114-124.

8. Marchioli R, Finazzi G, Specchia G, Cacciola R, Cavazzina R, Cilloni D, De Stefano V, et al. Cardiovascular events and intensity of treatment in polycythemia vera. N Engl J Med. 2013;368(1):22-33.

9. Barosi G, Birgegard G, Finazzi G, Griesshammer M, Harrison C, Hasselbalch H, Kiladijan JJ, et al. A unified definition of clinical resistance and intolerance to hydroxycarbamide in polycythaemia vera and primary myelofibrosis: results of a European LeukemiaNet (ELN) consensus process. Br J Haematol. 2010;148(6):961-963.

10. Barbui T, Tefferi A, Vannucchi AM, Passamonti F, Silver RT, Hoffman R, Verstovsek S, et al. Philadelphia chromosome-negative classical myeloproliferative neoplasms: revised management recommendations from European LeukemiaNet. Leukemia. 2018;32(5):1057-1069.

11. Harrison C, Kiladjian JJ, Al-Ali HK, Gisslinger H, Waltzman R, Stalbovskaya V, McQuitty M, et al. JAK inhibition with ruxolitinib versus best available therapy for myelofibrosis. N Engl J Med. 2012;366(9):787-798.

12. Tremblay D, Mascarenhas J. Novel therapies in polycythemia vera. Current hematologic malignancy reports. Myeloproliferative Neoplasms (B Stein, Section Editor). 2020: p. 1-8.

13. Verstovsek S, Vannucchi AM, Griesshammer M, Masszi T, Durrant S, Passamonti F, Harrison CN, et al. Ruxolitinib versus best available therapy in patients with polycythemia vera: 80 -week follow-up from the RESPONSE trial. Haematologica. 2016;101(7):821-829.

14. Sant'Antonio E, Bonifacio M, Breccia M, Rumi E. A journey through infectious risk associated with ruxolitinib. Br J Haematol. 2019;187(3):286-295.

15. Tremblay D, King A, Li L, Moshier E, Coltoff A, Koshy A, Kremyanskaya M, et al. Risk factors for infections and secondary malignancies in patients with a myeloproliferative neoplasm treated with ruxolitinib: a dual-center, propensity score-matched analysis. Leuk Lymphoma. 2020;61(3):660-667.

16. AIFA register. Available from: www.AIFA.gov.it. 